\title{
Actual availability of general practice appointments for mildly ill children
}

\section{Study question}

There are concerns that Australia's primary care workforce may be in shortage. Actual or perceived lack of availability of primary care appointments may contribute to the increased rates of attendances at hospital emergency departments (EDs). We undertook this study to assess actual same-day availability of appointments with general practitioners for mildly ill children.

\section{Methods}

We used "secret shopper" techniques to determine the actual availability of GP appointments. Phone calls were made to 225 general practice clinics, 75 from each of three Medicare Local catchment areas, by research assistants posing as parents seeking treatment for children with non-urgent, low-acuity conditions.

Same-day appointments were sought for paediatric patients based on one of two clinical scenarios designed to present non-urgent, low-severity conditions appropriate for general practice. The scenarios were developed with the help of two clerical staff from two general practice clinics and two GPs from other practices. The scenarios were:

Gary L Freed $B A, M D, M P H$

Amie Bingham $\mathrm{MPH}, \mathrm{BSC}, \mathrm{BA}^{\prime}$

Amy R Allen BSc(Hons), GradDipAlcohol OtherDrugStudies'

Michele Freed $^{2}$

Lena A Sanci MB BS, FRACGP, PhD'

Neil Spike MB BS, FRACGPI

1University of Melbourne, Melbourne, VIC.

2 University of Michigan, Ann Arbor, Mich, USA.

gary.freed@ unimelb.edu.au

This is a research article summary. The full-length article is available on mja.com.au:

doi: 10.5694/mjal4.01724 ment with a specific GP. If a same-day Medicare Local catchment areas appointment with that provider was not available, they requested a sameday appointment with any GP in the clinic. In clinics where same-day appointments were available, the time between making the call and the next available appointment was recorded. The bulk-billing status of the clinic was also recorded.

\section{Findings}

Overall, same-day appointments to see any doctor were offered by $78 \%$ of clinics (175/225). Across the three Medicare Local catchment areas, appointment availability varied only slightly, from $72 \%$ to $81 \%$. However, availability to see a specific doctor in the practice was more limited (Box). About half of the clinics contacted $(111 ; 49 \%)$ were able to offer an appointment within 4 hours. A further $9 \%$ offered walk-in appointments, for which a waiting time could not accurately be determined.

About three-quarters of the clinics contacted $(168 ; 75 \%)$ offered bulk-billing for mildly ill children. Appointments were more likely to be available in clinics that provided bulk billing for paediatric appointments, with $82 \%$ of those able to offer an appointment compared with $67 \%$ of fee-paying clinics $(P=0.01)$.

\section{Limitations}

The distribution of GPs across Australia is variable, so our findings may not be generalisable to other areas. Also, we collected data at a time likely to maximise appointment availability (weekday mornings). A similar audit conducted later in the day (closer to closing time) may produce different results.

\section{What this study adds to current knowledge}

We showed that there is substantial realtime, same-day GP appointment availability for children with low urgency, low acuity conditions in the areas studied. Further, about three-quarters of clinics with same-day appointment availability offered bulk billing for paediatric patients, suggesting that financial constraints are unlikely to be a significant barrier to accessibility of primary-care. With $78 \%$ of clinics able to offer a sameday appointment and with there being multiple GPs within the postcodes of our catchment areas, it seems likely that most community members who are attempting to procure such an appointment would be able to do so on the same day and with no out-of-pocket cost.

\section{Implications for practice or policy}

Our results indicate that increasing attendances for children at EDs are unlikely to be driven primarily by an actual lack of same-day availability of GP appointments. These findings are important for developing data-driven policy approaches to the development and use of primary health care. Public perception that GP appointments are not available may be a contributing factor in some ED presentations. The public should be made aware of the ready availability of GP appointments.

Competing interests: No relevant disclosures.

(c) 2015 AMPCo Pty Ltd. Produced with Elsevier B.V. All rights reserved.

Availability of same-day appointments for a mildly ill child in 225 general practices within three

\begin{tabular}{lcccc} 
& & \multicolumn{3}{c}{ Appointments available } \\
\cline { 2 - 5 } Medicare Local & $\begin{array}{c}\text { Total } \\
\text { phone calls }\end{array}$ & $\begin{array}{c}\text { With requested } \\
\text { general practitioner }\end{array}$ & $\begin{array}{c}\text { With another general } \\
\text { practitioner }\end{array}$ & Total \\
\hline NMML & 75 & $25(33 \%)$ & $36(48 \%)$ & $61(81 \%)$ \\
MRNWMML & 75 & $17(23 \%)$ & $43(57 \%)$ & $60(80 \%)$ \\
INWMML & 75 & $30(40 \%)$ & $24(32 \%)$ & $54(72 \%)$
\end{tabular}

NMML = Northern Melbourne Medicare Local (outer urban); MRNWMML = Macedon Ranges and North Western Melbourne Medicare Local (outer urban and regional); INWMML = Inner North West Melbourne Medicare Local (inner urban). 


\section{Actual availability of general practice appointments for mildly ill children}

There is
extensive
same-
day new-
patient GP
appointment
availability
for mildly ill
children in the
catchment
areas of
Melbourne
studied

$\mathrm{T}$ here are concerns that there may be shortages in the Australian primary care workforce, especially in rural and regional areas. However, some have suggested that a shortage of general practitioners is also a problem in metropolitan areas. ${ }^{1}$ In some outer suburbs of Melbourne, residents have reported significant difficulty in acquiring a medical certificate for sick leave in a timely manner, and that waiting 2 weeks for a doctor's appointment is common. ${ }^{2}$ Similarly, some GPs in Melbourne have reported having to close their books due to excessive demand, with one doctor stating he had to turn patients away for 2 years $^{3}$. Even in some inner-city Melbourne practices, doctors have reported having to close their books, and hearing from patients that they have had to telephone four or five clinics just to obtain an appointment. ${ }^{4}$

An actual or perceived lack of availability of primary care appointments may contribute to the increased rates of attendances at hospital emergency departments (EDs), as patients seek alternative ways to reach health care providers. Attendances at EDs are increasing at a rate exceeding population growth, and there is a perception among hospital staff that a significant portion of attendances are for conditions that do not require the urgent or specialised services that such departments provide., ${ }^{5,6}$ While some ED visits are for serious acute conditions some of which result in hospitalisation, a large number could be treated in a primary care setting. ${ }^{6-8}$

Many hospital EDs are experiencing a severe strain on their limited resources, as evidenced by chronic overcrowding and long waiting periods. ${ }^{9,10}$ Thus, ensuring that primary care practitioners are sufficiently available and accessible in the community is essential for both individuals and the health system as a whole. This would help patients with urgent conditions receive more timely

\section{Abstract}

Objective: To determine actual availability and cost of general practitioner appointments for children with conditions of low acuity and low urgency, from the perspective of the child's family.

Design, participants and setting: A "secret shopper" method, whereby research assistants posing as parents seeking appointments for mildly ill children telephoned a random sample of 225 general practice clinics within three Melbourne Medicare Local catchments, which included urban, suburban and regional areas; the study was conducted between 1 August and 30 September 2014.

Main outcome measures: Availability of same-day appointments and time until appointment; bulk-billing status of the clinic and/or the potential cost of an appointment.

Results: High availability of appointments was found in all three catchment areas (range, $72 \%-81 \%$ of clinics contacted). About half (49\%) had appointments available within 4 hours. Between $72 \%$ and $80 \%$ of clinics contacted in the three Medicare Local catchment areas offered bulk-billing for paediatric appointments.

Conclusion: There is extensive same-day new-patient GP appointment availability for mildly ill children in the catchment areas of Melbourne studied. Further, as most of the available appointments are in clinics that bulk bill, financial access should not affect this availability. Increased paediatric presentations to emergency departments are not likely to be the result of limited GP availability. These findings provide an important base for developing data-driven policy approaches to the development and use of primary care.

treatment in hospitals by reducing demand for ED services, while those who do not require emergency treatment would benefit from the continuity of care and preventive services available in the community. ${ }^{11}$

Understanding the true availability of the primary care workforce for the population is necessary to determine whether a shortage actually exists. Projected models of supply and demand for the primary care workforce do indicate a possible shortage of practitioners. ${ }^{12}$ However, beyond head counts of providers, there are few data that provide more than anecdotal reporting of primary care workforce supply and demand in Australia. For example, in 2009 the Australian Bureau of Statistics found that, in 1 year, about 937800 people reported they had been unable to access health services when they needed them. Of the $82 \%$ who were attempting to access general practice services, $47 \%$ cited long waiting periods or a lack of available appointments as the main reason for lack of access. ${ }^{13}$ A study of an outer metropolitan region in Western Australia found that $59 \%$ of people had reported difficulty in obtaining GP appointments, citing lack of appointments, long waiting periods and inconvenient hours. ${ }^{14}$ Although indicative, such data are of limited use in assessing the actual availability and accessibility of services, given their focus on modelled data or on patient recall of health service access. No previous studies have provided a real-time assessment of GP availability or appointment charges from the patient's perspective.

The lack of such data hinders adequate policy responses to ensure that the needs of the population are met. We undertook this study specifically to help fill this gap. We focused particularly on children because previous studies have shown a decrease in the proportion 
1 Availability of same-day appointments for a mildly ill child in 225 general practices within three Medicare Local catchment areas

\begin{tabular}{lcccc} 
& \multicolumn{3}{c}{ Appointments available } \\
\cline { 2 - 5 } Medicare Local & $\begin{array}{c}\text { Total } \\
\text { phone calls }\end{array}$ & $\begin{array}{c}\text { With requested } \\
\text { general practitioner }\end{array}$ & $\begin{array}{c}\text { With another general } \\
\text { practitioner }\end{array}$ & Total \\
\hline NMML & 75 & $25(33 \%)$ & $36(48 \%)$ & $61(81 \%)$ \\
MRNWMML & 75 & $17(23 \%)$ & $43(57 \%)$ & $60(80 \%)$ \\
INWMML & 75 & $30(40 \%)$ & $24(32 \%)$ & $54(72 \%)$
\end{tabular}

NMML = Northern Melbourne Medicare Local (outer urban); MRNWMML = Macedon Ranges and North Western Melbourne Medicare Local (outer urban and regional); INWMML = Inner North West Melbourne Medicare Local (inner urban).

of GP visits specifically provided to children over the past 2 decades. ${ }^{15}$

\section{Methods}

We used "secret shopper" techniques, a well known and often-used method to determine the actual versus reported availability of physician appointments. ${ }^{16}$ This involved having research assistants pose as parents and telephoning general practice clinics seeking treatment for children with non-urgent, low-acuity conditions. The study was conducted between 1 August and 30 September 2014.

\section{Sample}

The sampling frame included all GPs working within the catchment areas of three Medicare Locals in the greater Melbourne metropolitan area. The Medicare Local catchments included urban, suburban and regional areas. Each Medicare Local provided contact details of all GPs within their catchment areas. A random sample of 225 practices were contacted, 75 from each Medicare Local list.

\section{Data collection and analysis}

Same-day appointments were sought for paediatric patients based on one of two clinical scenarios, each of which was designed to present non-urgent, low-severity conditions appropriate for general practice. The scenarios were developed with the help of two clerical staff from two general practice clinics and two GPs from other practices. The scenarios were:

- a 3-year-old child with an earache and a mild fever; and

- an 8-month-old with a slight cough, runny nose and mild fever.

\section{Availability of appointments and time until appointment in 225 general practices}

\begin{tabular}{lccc}
$\begin{array}{l}\text { Time to } \\
\text { appointment } \\
\text { (hours) }\end{array}$ & No. & Proportion & $\begin{array}{c}\text { Cumulative } \\
\text { proportion }\end{array}$ \\
\cline { 2 - 4 } Within 1 & 34 & $15 \%$ & $15 \%$ \\
Within 2 & 38 & $17 \%$ & $32 \%$ \\
Within 3 & 26 & $12 \%$ & $44 \%$ \\
Within 4 & 13 & $6 \%$ & $49 \%$ \\
Within 5 & 20 & $9 \%$ & $58 \%$ \\
Within 6 & 13 & $6 \%$ & $64 \%$ \\
Within 7 & 6 & $3 \%$ & $67 \%$ \\
Within 8 & 4 & $2 \%$ & $68 \%$ \\
8 or more & 1 & $0 \%$ & $69 \%$ \\
Walk-in (time & 20 & $9 \%$ & $78 \%$ \\
unknown) & & & \\
No appointments & 50 & $22 \%$ & \\
available & & &
\end{tabular}

Research assistants telephoned clinics between 9:00 am and 12:00 pm on weekdays to maximise the likelihood of same-day appointment availability.

Attempts were made to make an appointment with a specific, randomly selected GP in the practice. If a same-day appointment with that GP was not available, a request was made for a same-day appointment with any GP in the clinic. Same-day appointments offered with other GPs within the clinic were classified as an available appointment. Data were recorded on whether an appointment was unavailable, available with the requested practitioner, or available with another practitioner within the clinic. Where same-day appointments were available, the time between making the call and the next available appointment was recorded. Bulk-billing status of the clinic was a binary variable (yes/ no). Where clinics did not bulk bill, both the upfront cost of an appointment and the final out-of-pocket cost (upfront cost minus the Medicare rebate amount) was recorded. Where the receptionist stated the cost of an appointment was likely to vary on length of appointment, a minimum and maximum cost provided by the practice were recorded.

Where an appointment was offered, the appointment itself was not accepted. Receptionists were told by the research assistant that they would call back if an appointment was actually needed and if the time offered was acceptable. No appointments were actually booked in any practice. The call process was developed and pilot-tested with medical reception staff from GP clinics to ensure that it did not have 
a significant impact on the administration of the clinics.

The study received ethics clearance from the University of Melbourne Human Research Ethics Committee. This committee required that, after the data were collected, letters be sent to clinics that had been selected within the catchment areas alerting them to the possibility of having been contacted by the research team and describing the nature of the study.

\section{Statistical analysis}

All data were analysed using Stata, version 13 (StataCorp). Analyses primarily involved the use of descriptive statistics, with the $\chi^{2}$ test used to investigate potential associations between appointment availability and the availability of bulk-billing in clinics.

\section{Results}

\section{Appointment availability}

Overall, same-day appointments to see any doctor were offered by $78 \%$ of clinics (175/225). Appointment availability between the three Medicare Local catchment areas varied only slightly, from $72 \%$ to $81 \%$. However, availability to see a specific doctor in the practice was more limited (Box 1).

Time until available appointments ranged from less than 1 hour in $15 \%$ of practices to over 8 hours in a single practice (Box 2). About half of the clinics contacted $(111 ; 49 \%)$ were able to offer an appointment within 4 hours. A further $9 \%$ offered walk-in appointments, for which a waiting time could not accurately be determined (Box 2).

\section{Appointment cost}

About three-quarters of the clinics contacted $(168 ; 75 \%)$ offered bulk-billing for paediatric patients. Actual proportions varied between $72 \%$ and $80 \%$ in the three Medicare Local catchment areas (Box 3).

Box 4 provides a comparison of appointment availability by clinic billing practices. Appointments were more likely to be available in clinics that provided bulk-billing for paediatric appointments, with $82 \%$ of those able to offer an appointment compared with $67 \%$ of private-billing clinics $(P=0.01)$. Mean out-of-pocket costs were lower at clinics in which appointments were available than at those in which no appointment was offered. In clinics that did not bulk bill, the minimum out-of-pocket costs cited by reception staff ranged from $\$ 2$ to $\$ 75.65$, with a mean of $\$ 32.18$. Maximum out-of-pocket costs ranged from $\$ 12$ to $\$ 94.30$, with a mean of $\$ 34.67$.

\section{Discussion}

Among the most important findings from this study is that there is significant same-day GP appointment availability for children with low urgency, low-acuity conditions in the areas we studied. Further, about three-quarters of clinics with sameday appointment availability offered bulk-billing for paediatric patients, suggesting that financial constraints are unlikely to be a significant barrier to accessibility of primary care appointments. With $78 \%$ of clinics able to offer a same-day appointment and with there being multiple GPs practising within the postcodes of our catchment areas, it seems likely that most people attempting to procure such an appointment would be able to do so, and with no

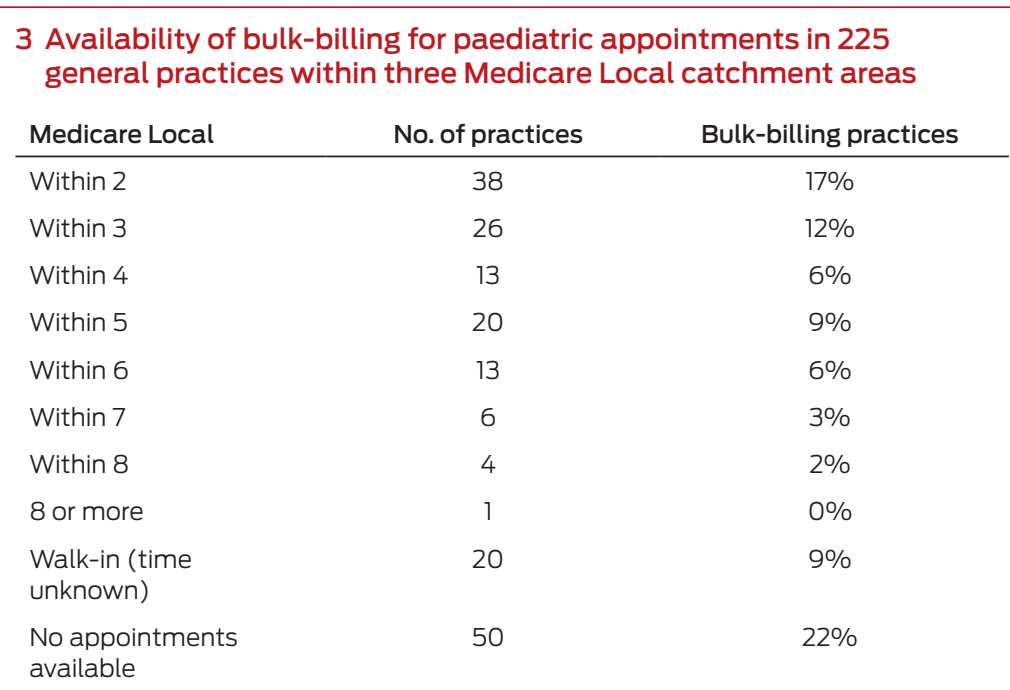

NMML = Northern Melbourne Medicare Local (outer urban); MRNWMML = Macedon Ranges and North Western Melbourne Medicare Local (outer urban and regional); INWML = Inner North West Melbourne Medicare Local (inner urban). $\bullet$ out-of-pocket cost. In addition, a high proportion (almost $60 \%$ ) of appointments were offered either within 4 hours of contacting the clinic or on a same-day walk-in basis.

Our results indicate that increasing attendances for children at EDs are unlikely to be driven primarily by a lack of availability of appointments in general practice. It is possible that a perceived lack of primary care appointment availability may be a contributing factor in some ED presentations. Parents and guardians may simply be assuming a lack of availability of GP appointments, and believe they are more likely to be provided with timely care in an ED. If this is the case, public information campaigns based on our findings may help to change the behaviour of patients in seeking acute care for low urgency conditions at their general practice clinic. Although not all GPs had same-day appointments available, there were multiple GPs able to see patients for non-urgent conditions in a timely manner. This is in contrast to the picture presented in some media reports. ${ }^{2-4}$

Our findings on the high proportion of clinics that bulk bill for paediatric care $(75 \%)$, and the higher availability of appointments in these clinics compared with private-billing clinics is important. The perceived cost of appointments may be influencing the behaviour of parents seeking 

4 Availability of and billing practices for paediatric appointments in
225 general practices

\begin{tabular}{lccc}
$\begin{array}{l}\text { Appointment } \\
\text { available }\end{array}$ & No. of practices & Bulk-billing & Private-billing \\
\hline No & 50 & $31(62 \%)$ & $19(38 \%)$ \\
Yes & 175 & $137(78 \%)$ & $38(22 \%)$ \\
All practices & 225 & 168 & 57
\end{tabular}

care for children with non-urgent conditions. ${ }^{17}$ A study of patients 15 years and older found that over a million Australians reported that they had not seen, or had delayed seeing, a doctor due to cost in a given year; another study found that $24.5 \%$ of those living in an outer metropolitan suburb cited financial matters as a barrier to visiting a GP. ${ }^{13,14}$ Publicising the availability and accessibility of same-day appointments for children in bulk-billing clinics may allay concerns over the cost of appointments.

A previous study has shown that if parents are unable to obtain appointments with a practitioner that they know and trust, they may instead seek treatment in an ED. ${ }^{18}$ Although parents may wish to see the same
GP for all of their child's health care needs, that appears to be an increasingly unrealistic goal. Trends in GP practices indicate that the number of GPs working in larger practices (of four or more partners) is increasing, while only about $11 \%$ work in solo practices. ${ }^{19-21}$ Frequently, patients are seen by several members of a practice.

A limitation of our study is that that the distribution of GPs may differ between metropolitan, regional and rural areas across the country, so our findings may not be generalisable to other parts of Australia. Further research would be required to assess the availability of same-day GP appointments in other areas. Further, we collected our data on weekday mornings - a time likely to maximise appointment availability. A similar audit conducted nearer closing time may produce different results.

This study provides an accurate and current real-time assessment of availability and accessibility of general practice appointments for children with non-urgent conditions from the patient's perspective. We found high availability of appointments in GP practices, although not necessarily with a specific doctor in the practice. Additionally, the high availability of appointments in bulk-billing clinics indicates a lack of economic barriers to available care. We believe this information can aid in developing data-driven policy approaches for ensuring the availability of the primary care workforce and the use of paediatric ED services for non-urgent conditions. Further, the public should be made aware of the ready availability of GP appointments.

Acknowledgements: We thank the Victorian Department of Health, Royal Children's Hospital Foundation, Inner North West Melbourne Medicare Local and Northern Melbourne Medicare Local for financial support for the project.

Competing interests: No relevant disclosures.

(c) 2015 AMPCo Pty Ltd. Produced with Elsevier B.V. All rights reserved. 
1 Strong K, Trickett P, Titulaer I, Bhatia K. Health in rural and remote Australia: the first report of the Australian Institute of Health and Welfare on rural Health. Canberra: AlHW, 1998. (AlHW Cat. No. PHE 6.) http://www.aihw.gov.au/publicationdetail/?id=6442466996 (accessed May 2015).

2 Hammond G. Price of living on the fringe. Sunday Herald Sun 2011; 18 Dec: 37.

3 David R, Clifton-Evans L. Doctors in demand in outer areas. Call for more GPs in suburbs to ease pain. Heidelberg Leader 2012; 14 Aug.

4 David R, Clifton-Evans L. Health wait: the queues are staying long. Call for more GPs in suburbs to ease pain. Bayside Leader. 201212 August 2012.

5 Masso M, Bezzina AJ, Siminski P, et al. Why patients attend emergency departments for conditions potentially appropriate for primary care: reasons given by patients and clinicians differ. Emerg Med Australas 2007; 19: 333340.

6 Lowthian JA, Curtis AJ, Jolley DJ, et al. Demand at the emergency department front door: 10-year trends in presentations. Med J Aust 2012; 196: 128-132. https://www.mja.com. au/journal/2012/196/2/demandemergency-department-front-door10-year-trends-presentations

7 Nagree Y, Mountain D, Cameron P, et al. Determining the true burden of general practice patients in the emergency department: the need for robust methodology. Emerg Med Australas 2011; 23: 116-119.

8 Nagree Y, Camarda VJ, Fatovich DM, et al. Quantifying the proportion of general practice and lowacuity patients in the emergency department. Med J Aust 2013; 198: 612-615. https://www.mja.com.au/ journal/2013/198/11/quantifyingproportion-general-practice-and-lowacuity-patients-emergency

9 Forero R, Hillman KM, McCarthy S, et al. Access block and ED overcrowding. Emerg Med Australas 2010; 22: 119-135.

10 Derlet RW. Overcrowding in emergency departments: increased demand and decreased capacity. Ann Emerg Med 2002; 39: 430-432.

11 Ben-Isaac E, Schrager SM, Keefer M, Chen AY. National profile of nonemergent pediatric emergency department visits. Pediatrics 2010; 125: 454-459.
12 Australian Medical Workforce Advisory Committee. The general practice workforce in Australia: supply and requirements to 2013. Sydney: AMWAC, 2005.

13 Australian Bureau of Statistics. Health services: patient experiences in Australia, 2009. Canberra: ABS, 2010 (ABS Cat. No. 4839.0.55.001.) http:// www.abs.gov.au/AUSSTATS/abs@. nsf/Lookup/4839.0.55.001Main+Featu resl2009?OpenDocument

14 McGaw A, Jayasuriya P, Bulsara C, Thompson SC. Accessing primary health care: a community survey of issues regarding general practice and emergency department services in an outer metropolitan area. Aust $J$ Primary Health 2007; 12: 78-84.

15 Freed GL, Sewell J, Spike N, et al. Changes in the demography of Australia and therefore general practice patient populations. Aust Fam Physician 2012; 41: 715.

16 Bisgaier J, Rhodes KV. Auditing access to specialty care for children with public insurance. N Engl J Med 2011; 364: 2324-2333.

17 Williams A, O'Rourke P, Keogh S. Making choices: why parents present to the emergency department for non-urgent care. Arch Dis Child 2009; 94: 817-820.

18 Woolfenden S, Ritchie J, Hanson R, Nossar V. Parental use of a paediatric emergency department as an ambulatory care service. Aust N Z J Public Health 2000; 24: 204-206.

19 Britt H, Miller G, Charles J, et al. General practice activity in Australia, 2008-09. Canberra: Australian Institute of Health and Welfare, 2009 (AlHW Cat. No. GEP25.) http://www.aihw.gov.au/ publication-detail/?id=6442468308 (accessed May 2015).

20 Charles J, Britt $\mathrm{H}$, Valenti L. The evolution of the general practice workforce in Australia, 1991-2003. Med J Aust 2004; 181: 85-90. https://www. mja.com.au/journal/2004/181/2/ evolution-general-practice-workforceaustralia-1991-2003

21 Britt H, Miller G, Charles J, et al. A decade of Australian general practice activity 2002-03 to 2011-12. General Practice Series No. 32. Sydney: Sydney University Press, 2012. 